\title{
ChemComm
}

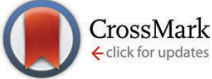

Cite this: Chem. Commun., 2015, 51,16417

Received 15th July 2015

Accepted 17th September 2015

DOI: $10.1039 / \mathrm{c} 5 \mathrm{cc} 05875 a$

www.rsc.org/chemcomm

\section{The construction of a two-dimensional supramolecular organic framework with parallelogram pores and stepwise fluorescence enhancement $\dagger$}

\author{
Shun-Qi Xu, Xiang Zhang, Cheng-Bin Nie, Zhong-Fu Pang, Xiao-Na Xu and \\ Xin Zhao*
}

\begin{abstract}
A novel single-layer two-dimensional (2D) supramolecular organic framework (SOF) with parallelogram pores has been assembled to turn on the fluorescence emission of a non-emissive building block, and the emission could be further enhanced by the aggregation of the as-prepared 2D monolayers.
\end{abstract}

Inspired by the discovery of graphene, in recent years twodimensional (2D) materials have become one of the most attractive subjects in materials science. ${ }^{1}$ Although currently many efforts have been devoted to the development of effective methods to construct 2D structures, the fabrication of this type of material has still been a great challenge. ${ }^{2}$ Very recently we reported the construction of the first single-layer 2D supramolecular organic framework (SOF) in water through cucurbit[8]uril (CB[8])-based host-guest chemistry. ${ }^{3}$ Lately we demonstrated that such 2D structures could have a significant impact on the properties of soft materials through the fabrication of highly thermally stable hydrogels from 2D SOFs. ${ }^{4}$ Due to their structural similarity to graphene, artificial 2D materials are also expected to display some intriguing features. However, the exploration of such features has been quite rare to date, as a result of the limited access to 2D structures.

Since the pioneering work of Tang and co-workers, ${ }^{5}$ aggregationinduced emission (AIE) or aggregation-induced enhanced emission (AIEE) has drawn considerable attention in the past decade because of their important applications in the fabrication of optical materials. ${ }^{6}$ The mechanism for the generation of AIE or AIEE is generally accepted to be the restriction of intramolecular rotation (RIR) of luminophores when they exist in the aggregating state. $^{7}$ On the basis of this mechanism, a variety of materials which exhibit AIE or AIEE phenomenon have been designed and fabricated, ${ }^{8}$ among which tetraphenylethene (TPE) is the most widely used scaffold. In the construction of AIE/AIEE-based

Key Laboratory of Synthetic and Self-Assembly Chemistry for Organic Functional Molecules, Shanghai Institute of Organic Chemistry, Chinese Academy of Sciences, 345 Lingling Road, Shanghai 200032, China. E-mail: xzhao@mail.sioc.ac.cn $\dagger$ Electronic supplementary information (ESI) available: Synthesis and characterization, Job's plot, DLS profiles, and additional fluorescence spectra. See DOI: 10.1039/ c5cc05875a materials, self-assembly has been demonstrated to be an efficient tool to realize RIR through non-covalent interactions. ${ }^{9}$ In this context, self-assembled 2D structures should have the very advantage to confine luminophores in $2 \mathrm{D}$ space and thus should have great potential to facilitate AIE or AIEE. However, to the best of our knowledge, such a phenomenon has never been observed in a real 2D structure before. In this communication, we report the construction of a TPE-based 2D SOF with parallelogram pores. AIE was observed when the non-emissive monomers co-assembled with $\mathrm{CB}[8]$ to form a single-layer $2 \mathrm{D}$ SOF in water (Scheme 1). Its fluorescence was further enhanced when the monolayers were pushed to aggregate. The stepwise emission enhancement demonstrates interesting AIE and AIEE phenomena originating from the unique 2D SOF structure.

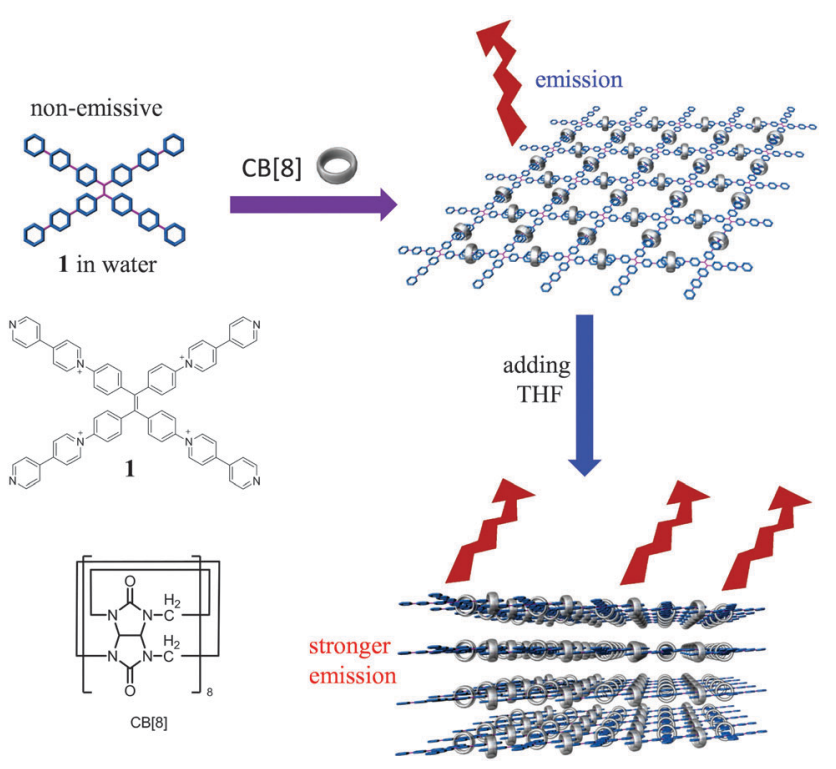

Scheme 1 The chemical structures of compound 1 and $C B[8]$ and cartoon representation for the formation of a $2 D$ SOF and stepwise fluorescence enhancement. 
The TPE-based building block (1) was designed by incorporating four 4,4'-bipyridin-1-ium (BP) units on the periphery of a TPE skeleton. We and Liu et al. have previously proved that $\mathrm{CB}[8]$ could encapsulate two BPs in its cavity in a head-to-tail arrangement so that supramolecularly polymeric structures could be assembled from $\mathrm{CB}[8]$ and rationally designed monomers. ${ }^{3,10}$ Directed by the preorganized conformation of the building block and the hostguest interaction, it is expected that a $2 \mathrm{D}$ SOF with parallelogram pores could be assembled from 1 and $\mathrm{CB}[8]$. The ${ }^{1} \mathrm{H}$ NMR titration experiment was firstly performed to confirm the formation of polymeric structures. As shown in Fig. 1, the addition of $\mathrm{CB}[8]$ to a solution of 1 in $\mathrm{D}_{2} \mathrm{O}$ resulted in the decrease of intensity and downfield shifts (except $\mathrm{H}_{1}$ and $\mathrm{H}_{2}$ ) of its peaks. The addition of 2.0 equiv. of $\mathrm{CB}[8]$ caused the signals of 1 to disappear completely. This result suggested that high-molecular-weight polymeric species formed in solution. Job's plot was further generated using UV-vis spectroscopy. It displayed a maximum absorption change at $33.3 \%$, indicating a $1: 2$ stoichiometry for 1 and $\mathrm{CB}[8]$ (Fig. S1, ESI $\dagger$ ).

The formation of large polymeric structures was further evidenced by the dynamic light scattering (DLS) experiment, which revealed that the hydrodynamic diameter $\left(D_{\mathrm{h}}\right)$ of the solution of the mixture of 1 and $\mathrm{CB}[8]$ (1:2) in water was $44.7 \mathrm{~nm}$ when the concentration of 1 was $0.25 \mathrm{mM}$ (Fig. S2, ESI $\dagger$ ). This value was 20 times larger than that of $\mathbf{1}$ alone in water at the same concentration. Concentration-dependence of $D_{\mathrm{h}}$ was also examined. It was found that the $D_{\mathrm{h}}$ value of the solution of the mixture of 1 and $\mathrm{CB}[8](1: 2)$ in water increased with the increasing concentration of the mixture (Fig. S2, ESI $\dagger$ ), indicating a higher degree of polymerization at higher concentration, which is a typical feature of supramolecular polymers.

The morphology of the as-prepared material was then visualized using microscopes. The transmission electron microscopy (TEM) image revealed the presence of film-like structures with some corrugations, suggesting the formation of extremely thin $2 \mathrm{D}$ structures (Fig. 2a). The thickness of the films was measured by means of atomic force microscopy (AFM). AFM also revealed the formation of flat and uniform films with a height of about $1.7 \mathrm{~nm}$, as indicated by cross-sectional analysis (Fig. 2b). This value agreed

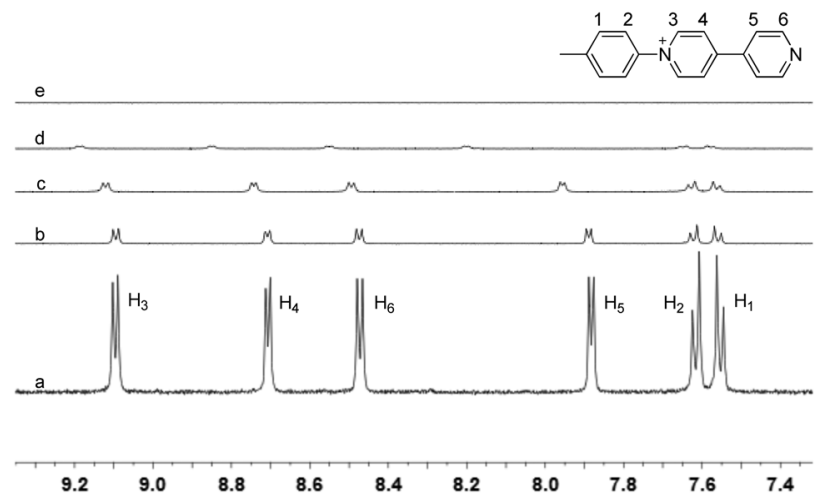

Fig. 1 Partial ${ }^{1} \mathrm{H}$ NMR spectra of (a) $\mathbf{1}$, (b) $\mathbf{1}+\mathrm{CB}[8](1: 0.2)$, (c) $\mathbf{1}+\mathrm{CB}[8]$ (1:0.5), (d) $1+C B[8](1: 1)$, and (e) $1+C B[8](1: 2)$ in $\mathrm{D}_{2} \mathrm{O}$ at $25^{\circ} \mathrm{C}$. The concentration of 1 was $0.25 \mathrm{mM}$.
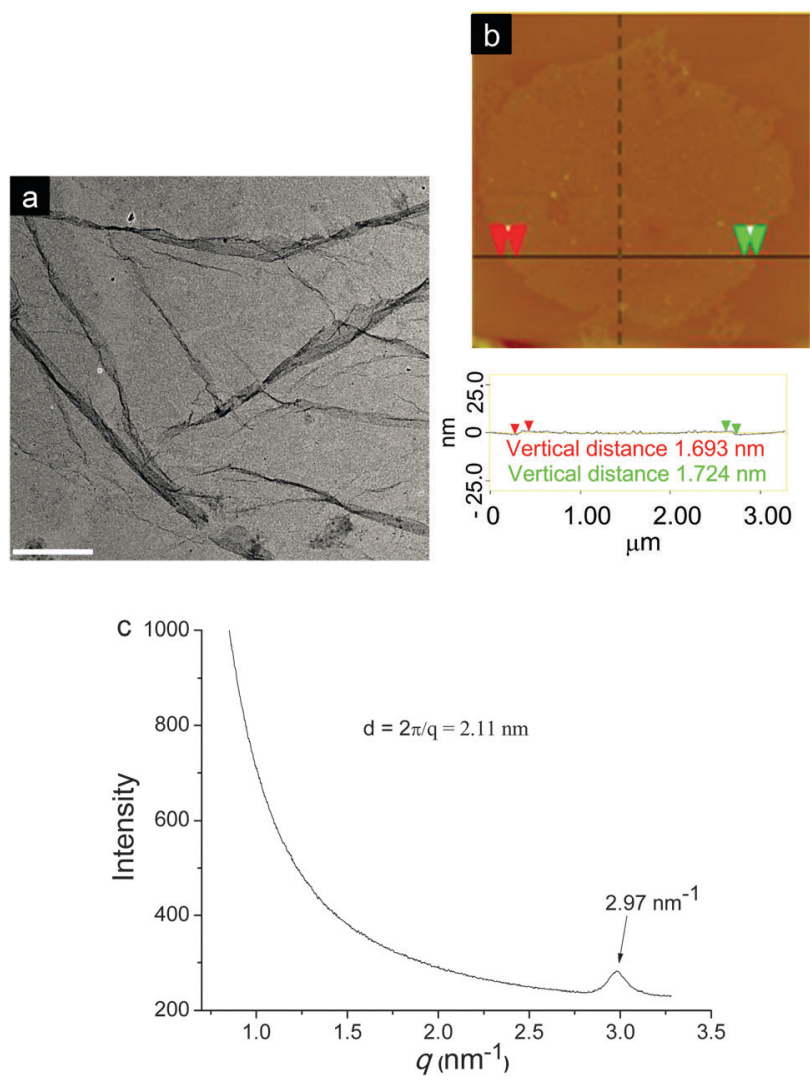

Fig. 2 (a) TEM image of the SOF fabricated from $1(0.05 \mathrm{mM})$ and $C B[8]$ (0.1 mM), (b) tapping-mode AFM image and section analysis of the SOF fabricated from $1(0.01 \mathrm{mM})$ and $C B[8](0.02 \mathrm{mM})$, and (c) synchrotron SAXD profile of the SOF obtained by evaporating a solution of $1(0.25 \mathrm{mM})$ and $\mathrm{CB}[8](0.5 \mathrm{mM})$ in water. The scale bar in the TEM image was $2 \mu \mathrm{m}$.

well with the diameter of rigid $\mathrm{CB}[8](1.75 \mathrm{~nm})$, confirming the formation of single-layer 2D SOFs.

In order to investigate the internal order of the as-formed $2 \mathrm{D}$ SOF which is a key criterion for a true 2D polymeric structure, the synchrotron small angle X-ray scattering (SAXS) experiment was carried out for the sample prepared by evaporating the solvent of an aqueous solution of 1 and $\mathrm{CB}[8](1: 2)$. SAXS displayed a scattering peak corresponding to a $d$ spacing of $2.11 \mathrm{~nm}$ (Fig. 2c). This value was well consistent with the theoretical pore width of the expected parallelogram pores $(2.08 \mathrm{~nm})$ of the SOF calculated using semi-empirical calculations, further confirming the formation of $2 \mathrm{D}$ SOF with periodic pore distribution.

After the structure of the 2D SOF was corroborated, its properties were then investigated. Since in this 2D SOF the TPE units were confined in $2 \mathrm{D}$ space, we anticipated that the AIE effect might be generated in this unique architecture. As expected, monomer $\mathbf{1}$ was non-emissive in aqueous solution (Fig. 3a). However, upon addition of $\mathrm{CB}[8]$ into the solution of $\mathbf{1}$, fluorescence emission could be detected. The intensity of emission increased with the increasing amount of $\mathrm{CB}[8]$ and reached a maximum when 2.0 equiv. of $\mathrm{CB}[8]$ was added (Fig. 3a). This fluorescence emission turn-on phenomenon could be attributed to the formation of the single-layer 2D SOF, in which free rotation of 

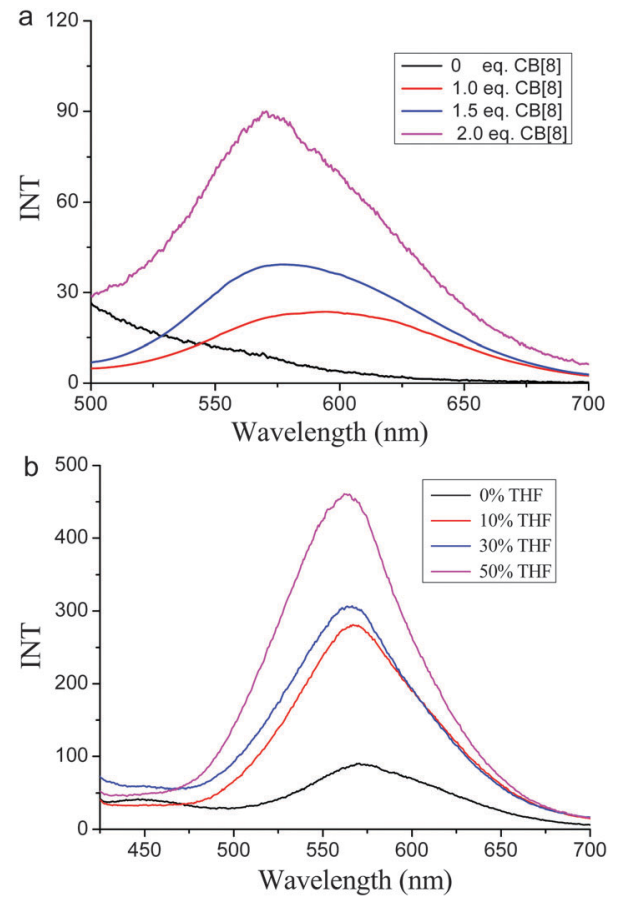

Fig. 3 Fluorescence spectral changes of (a) 1 (0.01 mM) upon gradual addition of $\mathrm{CB}[8]$ in water, and (b) fluorescence spectra of a SOF in waterTHF at different water $/ \mathrm{THF}$ ratios. $\lambda_{\mathrm{ex}}=360 \mathrm{~nm}$, Ex slit $=5 \mathrm{~nm}$, and Em slit $=$ $5 \mathrm{~nm}$. The SOF was fabricated from $1(0.01 \mathrm{mM})$ and $\mathrm{CB}[8](0.02 \mathrm{mM})$.

the TPE units was restricted and thus the radiative channel was opened.

A typical feature of $2 \mathrm{D}$ architectures is that they are prone to aggregate to form layered structures, as a result of the large surface areas of sheet-like structures. Since the SOF is an ionic species, solvophobic interactions should arise between the SOF and the organic solvents in which SOF cannot be well solvated. We anticipated that the addition of organic solvents with low and medium polarity should push the as-prepared single-layer 2D SOF to aggregate in the solution phase to reduce their contacting areas with solvent molecules. In such a way the unfavourable solvophobic interactions could be alleviated. Water-miscible organic solvents such as acetone, acetonitrile, dioxane, and tetrahydrofuran (THF) were examined by introducing them into the solution of 2D SOF in water. Fluorescence emission of the resulting solutions was then recorded, which revealed that the addition of these organic solvents to the aqueous solution of SOF all resulted in enhancement of its fluorescence emission at different levels (Fig. S3, ESI $\dagger$ ). Among the organic solvents tested, TFH did the best job, which should be attributed to its lowest polarity. Therefore, THF was selected as the poor solvent and more THF was further added. It was found that the fluorescence emission of the SOF remarkably increased upon the increased amount of THF (Fig. 3b). TEM investigation revealed that the 2D structure was still maintained even when $50 \%$ (v/v) THF was added (Fig. 4a). Moreover, the stacked layers could be observed from the TEM image, clearly indicating the aggregation of single-layer 2D SOFs. The DLS study confirmed again the aggregation of the monolayers in the presence of THF by demonstrating an increase of the
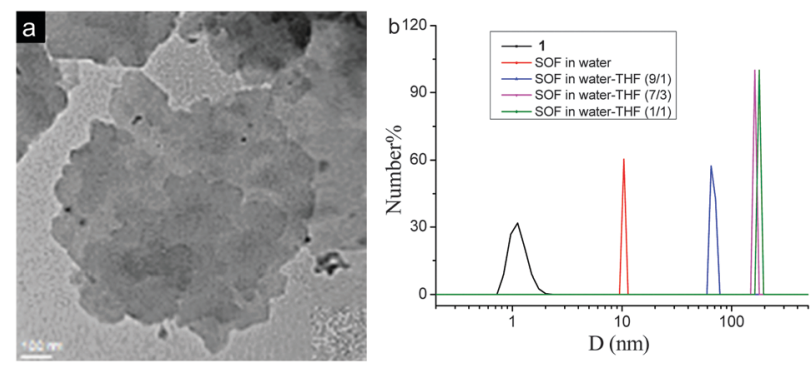

Fig. 4 (a) TEM image of the sample from $1(0.01 \mathrm{mM})$ and $C B[8](0.02 \mathrm{mM})$ in water-THF $(1: 1, V / V)$, and (b) DLS profiles of the solutions of the mixture $1(0.01 \mathrm{mM})$ and $\mathrm{CB}[8](0.02 \mathrm{mM})$ in water-THF at different water/THF ratios. The scale bar of the TEM image was $100 \mathrm{~nm}$.

hydrodynamic diameter of the species in the solution of $\mathbf{1}$ and $\mathrm{CB}[8](1: 2)$ with the increasing THF/water ratio of the binary solvent (Fig. 4b). Therefore, the enhancement of fluorescence emission of the 2D SOF after the addition of THF could be attributed to the aggregation of the single-layer SOF, which resulted in further inhibition of the rotation of TPE units in the stacked layers. In order to collect more evidence for this mechanism, a control experiment was performed by adding THF to aqueous solutions of $\mathbf{1}$ in the absence of $\mathrm{CB}[8]$. It was found that the intensity of fluorescence emission of $\mathbf{1}$ could also be enhanced, but just to a small extent (Fig. S4, ESI $\dagger$ ). This control experiment strongly suggested that it was the aggregation of a single-layer $2 \mathrm{D}$ SOF that further increased the fluorescence emission.

In summary, a novel water soluble single-layer two-dimensional supramolecular organic framework with parallelogram pores has been constructed through the assembly of $\mathrm{CB}[8]$ and a TPE-based building block. Thanks to the unique features of the TPE skeleton and the rigid 2D polymeric structure, fluorescence emission of the non-emissive building block was turned on after it co-assembled with $\mathrm{CB}[8]$ to form a single-layer 2D SOF. By taking the advantage of the sheet-like structure of the 2D SOF, they could be further pushed to aggregate and thus the fluorescence emission could be further enhanced. This study demonstrates that interesting optical properties can be derived from intriguing 2D structures, which make such structures highly promising for the fabrication of novel 2D functional materials.

We thank the National Natural Science Foundation of China (No. 91127007, 21402228, and 21472225) for financial support and Shanghai Synchrotron Radiation Facility for providing BL16B1 beamline for collecting the synchrotron X-ray scattering data.

\section{Notes and references}

1 R. Mas-Ballesté, C. Gómez-Navarro, J. Gómez-Herrero and F. Zamora, Nanoscale, 2011, 3, 20; J. Sakamoto, J. V. Heijst, O. Lukin and A. D. Schlüter, Angew. Chem., Int. Ed., 2009, 48, 1030; S.-L. Cai, W.-G. Zhang, R. N. Zuckermann, Z.-T. Li, X. Zhao and Y. Liu, Adv. Mater., 2015, DOI: 10.1002/adma. 201500124.

2 T.-Y. Zhou, F. Lin, Z.-T. Li and X. Zhao, Macromolecules, 2013, 46, 7745; K. Baek, G. Yun, Y. Kim, D. Kim, R. Hota, I. Hwang, D. Xu, Y. H. Ko, G. H. Gu, J. H. Suh, C. G. Park, B. J. Sung and K. Kim, J. Am. Chem. Soc., 2013, 135, 6523; K. S. Novoselov, D. Jiang, F. Schedin, T. J. Booth, V. V. Khotkevich, S. V. Morozov and A. K. Geim, Proc. Natl. Acad. Sci. U. S. A., 2005, 102, 10451; P. Kissel, R. Erni, W. B. Schweizer, M. D. Rossell, B. T. King, T. Bauer, S. Gotzinger, A. D. Schlüter and J. Sakamoto, 
Nat. Chem., 2012, 4, 287; P. Kissel, D. J. Murray, W. J. Wulftange, V. J. Catalano and B. T. King, Nat. Chem., 2014, 6, 774; M. J. Kory, M. Wörle, T. Weber, P. Payamyar, S. W. van de Poll, J. Dshemuchadse, N. Trapp and A. D. Schlüter, Nat. Chem., 2014, 6, 779.

3 K.-D. Zhang, J. Tian, D. Hanifi, Y. Zhang, A. C.-H. Sue, T.-Y. Zhou, L. Zhang, X. Zhao, Y. Liu and Z.-T. Li, J. Am. Chem. Soc., 2013, 135, 17913, For example of SOF in the solid state, see: J. Lü, C. PerezKrap, M. Suyetin, N. H. Alsmail, Y. Yan, S. Yang, W. Lewis, E. Bichoutskaia, C. C. Tang, A. J. Blake, R. Cao and M. Schröder, J. Am. Chem. Soc., 2014, 136, 12828.

4 T.-Y. Zhou, Q.-Y. Qi, Q.-L. Zhao, J. Fu, Y. Liu, Z. Ma and X. Zhao, Polym. Chem., 2015, 6, 3018.

5 J. Luo, Z. Xie, J. W. Y. Lam, L. Cheng, H. Chen, C. Qiu, H. S. Kwok, X. Zhan, Y. Liu, D. Zhu and B. Z. Tang, Chem. Commun., 2001, 1740; G. Liang, J. W. Y. Lam, W. Qin, J. Li, N. Xie and B. Z. Tang, Chem. Commun., 2014, 50, 1725; S. Zhang, J.-M. Yan, A.-J. Qin, J.-Z. Sun and B.-Z. Tang, Chin. Chem. Lett., 2013, 24, 668; H. Shi, R. T. K. Kwok, J. Liu, B. Xing, B. Z. Tang and B. Liu, J. Am. Chem. Soc., 2012, 134, 17972; D. Ding, K. Li, B. Liu and B. Z. Tang, Acc. Chem. Res., 2013, 46, 2441.

6 Y. Liu, S. M. Chen, J. W. Y. Lam, P. Lu, R. T. K. Kwok, F. Mahtab, H. S. Kwok and B. Z. Tang, Chem. Mater., 2011, 23, 2536; Y. Liu, C. Deng, L. Tang, A. Qin, R. Hu, J. Z. Sun and B. Z. Tang, J. Am. Chem. Soc., 2011, 133, 660; Z. Chang, Y. Jiang, B. He, J. Chen, Z. Yang, P. Lu, H. S. Kwok, Z. Zhao, H. Qiu and B. Z. Tang, Chem. Commun., 2013, 49, 594; X. Du, J. Qi, Z. Zhang, D. Ma and Z. Y. Wang, Chem. Mater., 2012, 24, 2178.
7 J. Mei, Y. Hong, J. W. Y. Lam, A. Qin, Y. Tang and B. Z. Tang, Adv. Mater., 2014, 26, 5429.

8 N. B. Shustova, B. D. McCarthy and M. Dincă, J. Am. Chem. Soc., 2011, 133, 20126; Z. Wei, Z.-Y. Gu, R. K. Arvapally, Y.-P. Chen, R. N. McDougald, Jr., J. F. Ivy, A. A. Yakovenko, D. Feng, M. A. Omary and H.-C. Zhou, J. Am. Chem. Soc., 2014, 136, 8269; Y. Xu, L. Chen, Z. Guo, A. Nagai and D. Jiang, J. Am. Chem. Soc., 2011, 133, 17622; Q. Chen, J.-X. Wang, F. Yang, D. Zhou, N. Bian, X.-J. Zhang, C.-G. Yan and B.-H. Han, J. Mater. Chem., 2011, 21, 13554; S. Ma, J. Zhang, J. Chen, L. Wang, B. Xu and W. Tian, Chin. J. Chem., 2013, 31, 1418; V. M. Suresh, S. Bonakala, S. Roy, S. Balasubramanian and T. K. Maji, J. Phys. Chem. C, 2014, 118, 24369.

9 G. Yu, G. Tang and F. Huang, J. Mater. Chem. C, 2014, 2, 6609; J. Wu, S. Sun, X. Feng, J. Shi, X.-Y. Hu and L. Wang, Chem. Commun., 2014, 50, 9122; J. Zhao, D. Yang, Y. Zhao, X.-J. Yang, Y.-Y. Wang and B. Wu, Angew. Chem., Int. Ed., 2014, 53, 1; P. Wang, X. Yan and F. Huang, Chem. Commun., 2014, 50, 5017; X. Yao, X. Ma and H. Tian, J. Mater. Chem. C, 2014, 2, 5155; X. Wang, J. Hu, T. Liu, G. Zhang and S. Liu, J. Mater. Chem., 2012, 22, 8622; N. Song, D.-X. Chen, Y.-C. Qiu, X.-Y. Yang, B. Xu, W. Tian and Y.-W. Yang, Chem. Commun., 2014, 50, 8231; R. Chen, H. Jiang, H. Gu, Q. Zhou, J. Wu, D. Chen and J. Zhang, Chem. Commun., 2015, 51, 12220; M. V. R. Raju and H.-C. Lin, Org. Lett., 2014, 16, 5564.

10 Z.-J. Zhang, H.-Y. Zhang, L. Chen and Y. Liu, J. Org. Chem., 2011, 76, 8270 . 